\title{
De anima ii 5 on the Activation of the Senses
}

\author{
John Bowin
}

Myles Burnyeat has argued that when at De anima ii 5.416b32-34, Aristotle

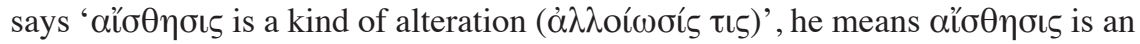
alteration in only a very restricted and etiolated sense-in the sense of a 'quasialteration' (Burnyeat 2002). A 'quasi-alteration' claims Burnyeat, is an alteration that can only be described in psychological or perceptual terms; it is to be identified with the process of becoming aware of an object of perception and as such it is not a 'literal' alteration of the sort that ordinary objects undergo. I argue, however, that one need not infer from what Aristotle says in De anima ii 5 that 'quasi-alteration' must be thought of in this way. Burnyeat's thesis, basically, is that Aristotle refines the notion of alteration in De anima ii 5 so that it picks out only perceptual alterations. But I establish that the 'refinement' Burnyeat attributes to Aristotle is either self-contradictory, and so picks out no alterations, or is so unrestrictive that it does not distinguish quasi-alterations from alterations in general. I suggest a fix for Burnyeat's refinement, based on a passage in Metaphysics $\mathrm{x} 5$, that renders it coherent and appropriately restrictive but consistent with non-perceptual alterations. I will then argue, based on what Aristotle says elsewhere in the De anima about affections of the sense organs and sense media, that even if quasi-alterations are alterations in some etiolated and non-literal sense, they still admit description in non-perceptual terms. If quasi-alterations are perceptual, then, they are evidently not perceptual in a way that precludes their being ordinary alterations.

The activation of the sense faculty

Much of the modern scholarship on De anima ii 5 has justly centered on the

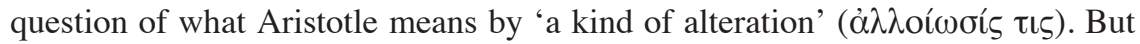

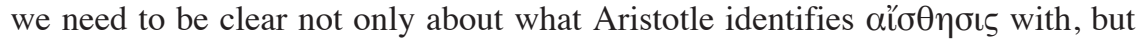
also about what he means by alø $\theta \eta \sigma \iota \varsigma$, since the word can have an unusually

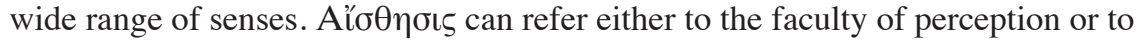
the organ of perception, or to perception as a kind of psychological event. ${ }^{1}$ When denoting a psychological event, as it appears to do in this chapter, Hamlyn notes that alı $\theta \eta \sigma ı \varsigma$ has a further ambiguity, since it can mean either the perception of a sense object or the perception that something is the case. Since the latter

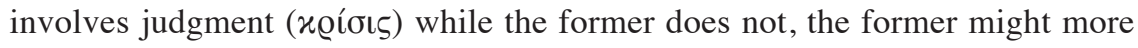

${ }^{1}$ See the notes on $417 \mathrm{a} 3$ and a12 in Hicks 1907, 351-352. 
accurately be called 'sensation' than 'perception'. Since De anima ii 5 treats per-

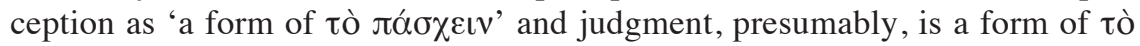

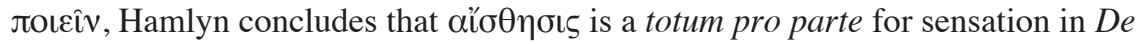
anima ii $5 .^{2}$ So when Aristotle says 'let us discuss generally the whole of $\alpha{ }^{\prime} \sigma \theta \eta \sigma ı \varsigma^{\prime}$ at the beginning of the chapter, he is inviting us to consider the most basic function of all the senses, not all the functions of the perceptive faculty. Burnyeat 2002, 33 seems to recognize this too, when he says 'how much information about the world [the senses] can deliver and in what form it comes' will be taken up later in the De anima and in the Parva Naturalia. What is at issue in this chapter is 'in what manner the senses operate [and] what explains their operation' (cf. Burnyeat 1995b, 428).

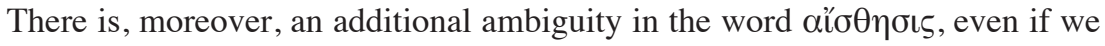

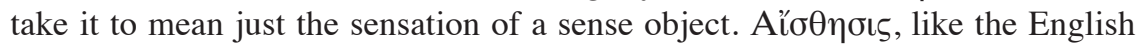
word 'sensation', can refer to either the activity of the sense faculty or to the process of making the sense faculty active, i.e., its activation by the agency of a sense object. Burnyeat and a growing number of other commentators have

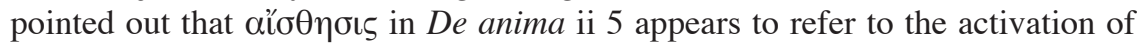
the sense faculty, i.e., to the transition to the activity of the sense faculty, not to the activity of the sense faculty itself. ${ }^{3}$ Though usually left unstated, what motivates this observation is apparently two assumptions: first, that we can infer what De anima ii 5 means by $\alpha$ ľ $\sigma \eta \eta \sigma \varsigma$ from the kind of alteration that Aristotle takes $\alpha$ ' $\sigma \theta \eta \sigma \iota \varsigma$ to be when he says ' $\alpha$ lı $\sigma \theta \eta \sigma \iota \varsigma$ is a kind of alteration', and second, that the kind alteration in question is what is described at 417a32-b2 as the activation of a natural disposition or faculty, such as a potential knower undergoes when he passes from a state of having knowledge to exercising it. The first assumption, I take it, is uncontroversial, and if De anima ii 5 does classify d' $\sigma \theta \eta \sigma \iota \varsigma$ as an alteration of this sort, then the sense of the word, at least within the confines of this chapter, must be the transition to the activity of the sense faculty. The truth of the second assumption, though less obvious, can be established by disjunctive syllogism, provided we can assume that the type of alteration to be identified with dí $\theta \eta \sigma ı \varsigma$ can be found among the types of alteration distinguished in De anima ii 5 .

De anima ii 5 identifies five sorts of alteration in addition to the one just described, and sets these off by three separate distinctions. The first distinction,

${ }^{2}$ Hamlyn 1959, 8. Hamlyn concedes that 'There are a few passages in Bk. 2 of the De anima in which the word x@íoı (or its derivatives) is used in connexion with aesthesis (418a4, 422a21, 424a5)' but points out that 'there are many more in Bk. 3 (425b21, 426b10 ff., 427a20, 428a3, 431a8, 20, 432aI5)'. See also Miller 1999, 201-209, who argues that, in addition to the passive affection of the sense organ, there is also an act of discrimination that goes on in perception, and he cites Somn. 2.455a13-20 for the doctrine that discrimination goes on in the primary sense organ, i.e., the heart, rather than in the eye, ear, etc.

${ }^{3}$ See Burnyeat 2002, 72-73; Barnes 1971/72, 108; Sorabji 1995, 220; Sorabji 2001, 50-51; Caston 2004, 249; Lorenz 2007, 212. Cf. Aristotle, Protrepticus apud Iamblichus, Protrepticus 57.2-6 Pistelli: 'a sleeping man must be said to live because of an ability to transition into that change (

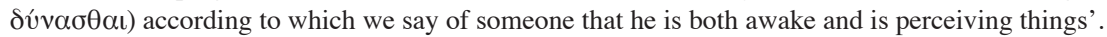


at 417a27-b2, opposes activations of natural dispositions and faculties to the acquisitions of these things, i.e., transitions such as from being able to know to knowing which a subject is able to undergo because his 'kind and matter' are of a certain sort (417a27, a31-32), or transitions such as from being able to acquire a sense faculty to possessing one, as in the case of a developing fetus (417b17). Let us follow Aristotle in calling these $\pi \varrho \omega ̂ \tau \alpha \iota ~ \mu \varepsilon \tau \alpha \beta o \lambda \alpha$ í or 'first transitions'. The second distinction contrasts destructive affections to preservative affections, or in Aristotle's words, affections involving 'a kind of destruction of something by its contrary' (417b2-3) to affections involving 'the preservation of that which is so potentially by that which is so actually' (417b3-5). The third distinction, at 417b14-16, distinguishes alterations 'toward privative conditions' from alterations 'toward a thing's dispositions and nature', e.g., learning. Let us call these 'privative alterations' and 'unordinary alterations' respectively. ${ }^{4}$ Of these five

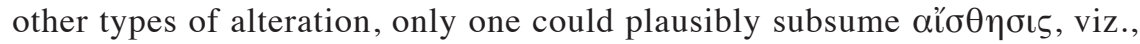
preservative affection. The others can be ruled out as the type of alteration Aristotle intends; first transitions and unordinary alterations because they apply to acquisitions of perceptual faculties rather than their exercise, destructive affections because they are contrasted with what are supposed to be analogous to

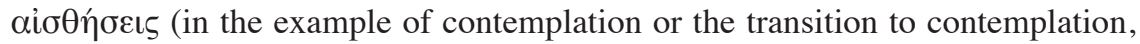
depending on how one reads 417b6-8), and privative alterations because alo $\theta \eta \sigma ı \varsigma$ under any interpretation is not a change to a privative condition. ${ }^{5}$ As for preservative affection, though dil $\sigma \eta \eta \sigma \iota \varsigma$ is clearly preservative in the sense that a sense faculty is preserved in its exercise, this category of change is much

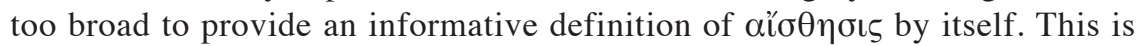
because, as Burnyeat 2002, 63 and 1984, 136, Gill 1989, 179, and Philoponus In $D A, 304,26-28$ have pointed out, acquisitions of dispositions and faculties that a subject is able to undergo because his 'kind and matter' are of a certain sort can also be described as affections involving 'the preservation of that which is so potentially by that which is so actually'. As Gill puts it, 'if a potentiality for knowledge belongs to an ignorant man because his genus and matter are appropriate, then the potentiality will still belong to him once he actually knows, because his matter and genus will still be suitable'. This suggests that when Aris-

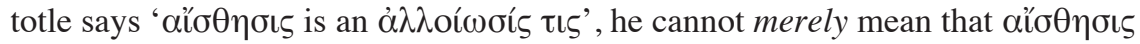

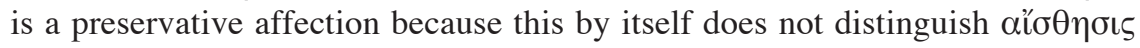
from the process of acquiring a perceptual faculty. Hence, by process of elimina-

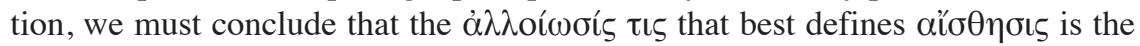

4 'Unordinary alterations' is Burnyeat's coinage (Burnyeat 2002, 65), and I adopt it here for the lack of a better word.

${ }^{5}$ Unordinary alterations are sometimes conflated with the activations of natural dispositions and faculties described at 417a32-b2 (see, e.g., Everson 1997, 89-96). But whereas the latter sort of transition is universally taken to represent a transition from a disposition or faculty to an activity, unordi-

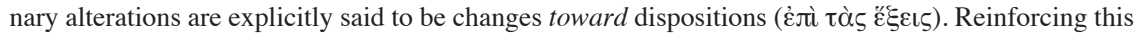
is the fact that Aristotle gives learning as an example of both unordinary alterations and first transitions, to which activations of natural dispositions and faculties are opposed (417b9-16). 
sort described at 417a32-b2, viz., the transition from having a natural disposition or faculty to using it, though this may be further described as a preservative affection (for a more detailed version of this argument, see Bowin 2011).

The failure to see that alo $\theta \eta \sigma i s$ refers to the activation of the sense faculty rather than its activity has been an impediment for many to see how it can be

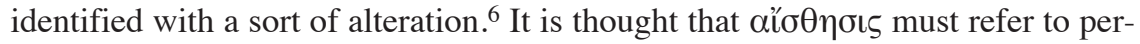
ceptual awareness, and as such, according to Metaphysics ix 6 and Nicomachean

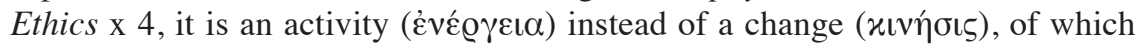
alteration $\left(\dot{\alpha} \lambda \lambda\right.$ oí $\left.^{\prime} \omega \sigma \iota \varsigma\right)$ is a species. The problem is that according to these two

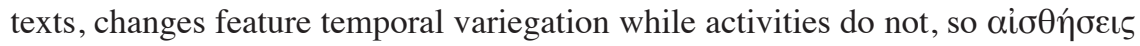

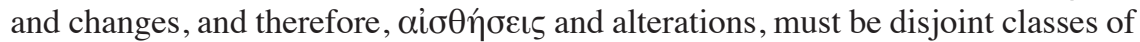
events. It then often assumed, as a consequence of this, that when Aristotle identifies al $\sigma \theta \eta \sigma \mathrm{s}$ with a sort of alteration, he must mean an alteration in a very strange and non-standard sense. But I do not detect a special interest in perceptual awareness in De anima ii $5 . .^{7}$ Of the words that usually signal such an inter-

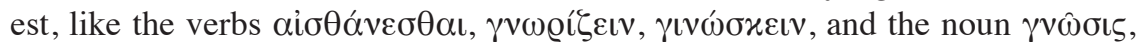
only ai $\sigma \theta \alpha \dot{v \varepsilon \sigma \theta} \theta \mathrm{u}$ appears in De anima ii 5, and its meaning is likely to track the

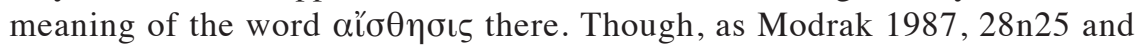
Burnyeat 1995a, 18 and 2002,33n12 note, Aristotle gives ample attention to the subjective experience of sensation elsewhere, De anima ii 5 appears to be a third person account of the transition to this state.

There are, moreover, at least two plausible explanations of why Aristotle is not concerned with the distinction between activity and change in De anima ii $5 .{ }^{8}$ The first one is Burnyeat's and supposes that Aristotle has an overriding interest in the cause of sensation in this chapter. Here, Aristotle wants to stress that the changes in a perceiver's sense organs that make him come to perceive are caused by a sense object, not by the perceiver himself (417b26-27). In other contexts such as Metaphysics ix 6 and Nicomachean Ethics x 4, where the cause of sensation is less important, this feature falls into the background and the 'transition to perceiving and perceiving can be allowed to merge' (Burnyeat 2002, 73). But for the time being, we are instructed to treat all actualities as incomplete, i.e., 'speak as if being affected, being moved, (

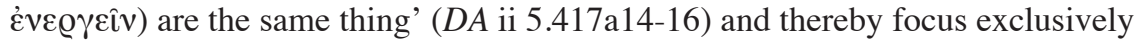
on the activation of the sense faculty rather than its activity.

Though this does have some plausibility, 417a14-16 could equally be taken to

${ }^{6}$ See Heinaman 1990, 92-93; Cohen 1995, 66; Everson 1997, 93n105, 255; Miller 1999, 197 200; Magee 2000, 318.

${ }^{7}$ Cf. Everson 1997, 172, who argues that Aristotle does not begin his discussion of perception 'by considering perceptual experience, but instead identifies perception by reference to its causes, the proper sensibles'.

${ }^{8}$ I say 'at least' because we can add, for instance, the explanation offered by Polansky 2007, 15. Polansky has argued that Aristotle refrains from invoking the distinction between xív $\dot{\varepsilon} v \varepsilon \varepsilon^{0} \gamma \varepsilon \iota \alpha$ on methodological grounds. The De anima, Polansky argues, studies the soul insofar as it is the nature of ensouled beings, and the nature of ensouled beings, according to Physics ii 1, is a princi-

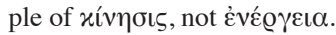


instruct us to disregard the distinction between complete and incomplete actualities, and this suggests a different account of why Aristotle is not concerned with

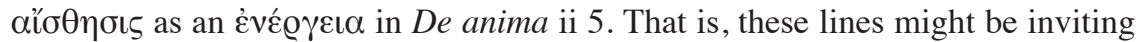
us to conflate the activity of the sense faculty with the transition to this activity,

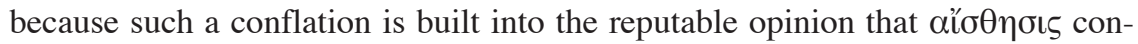
sists in being moved and affected. On anyone's account of change, and especially on Aristotle's, change cannot exist without temporal variegation. In Aristotle's case, this is because temporal variegation is necessary for difference in termini, and difference in termini is necessary for change (see, e.g., Phys. viii 7.261a3233 ). So to call a perceptual event an alteration is to imply that it is temporally variegated. It is, of course, true that in Metaphysics ix 6 and Nicomachean Ethics $\mathrm{x}$ 4, Aristotle denies temporal variegation to the activity of the sense faculty, and, indeed, to all activities, so on Aristotle's considered view, it is only the inception of sensation that has this feature. But to do justice to the reputable opinion that

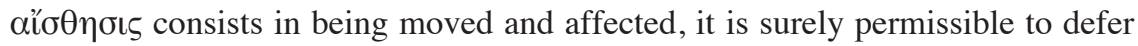
these refinements for the time being, and conflate the complete activity (i.e., tò

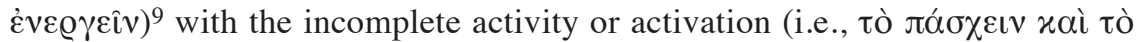

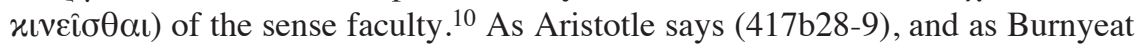
2002, 52, 66, 69 emphasizes, the discussion of perception in De anima ii 5 is incomplete. For now Aristotle is just concerned with a causal account of how sensory awareness arises, and is setting aside for another occasion other issues, such as perceptual content, judgment, the status of perception as an $\dot{\varepsilon} v \varepsilon \dot{\varepsilon} \gamma \varepsilon\llcorner\alpha$, and indeed, the nature of sensory awareness itself.

\section{Quasi-alteration}

I now examine in more detail what Aristotle identifies with the activation of

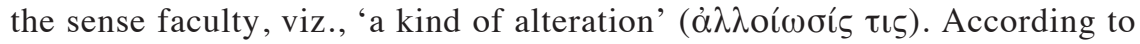
Burnyeat, Aristotle adapts, in De anima ii 5, the account of alteration from his physical works to meet the needs of his psychology. And Burnyeat's interpretation of how this account gets imported into De anima ii 5, at least in its initial stages (i.e., up to 417a20), is illuminating and highly plausible. Burnyeat argues that from the outset, the word $\alpha \lambda \lambda$ oí $\omega \sigma i \varsigma$ is theory-laden. It not only carries the technical Aristotelian sense of 'change in quality', but it also presupposes the

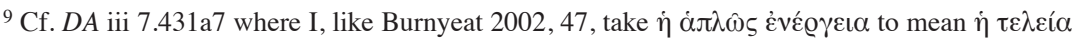

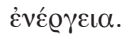

${ }^{10}$ This same conflation of activity and activation is also evident where the 'triple scheme' is employed at Phys. viii 4.255a30-b24. At one point in this passage, Aristotle says that the activity (غ่vÉ

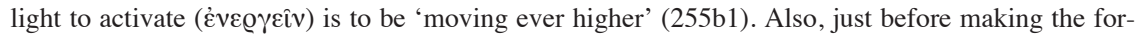

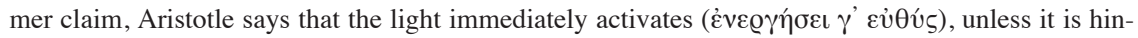
dered from doing so. But it is hard to see how this activity could be anything other than rising, if what is rising is any distance, however small, from being 'high up', because a period of rising would need to intervene before the light is 'high up', and this makes its being 'high up' not immediate. Thus, in Phys. viii 4.255a30-b24, both the activity of the light (being 'high up') and the activation of the light (moving 'high up') are referred to as activities of the light. 
doctrine of Generation and Corruption i 7 that to be altered is to be affected by something so as to become like it (324a10-11). So an alteration is at once an affection and an assimilation. The key thing to recognize, according to Burnyeat 2002, 41 is that the identification of perception with a kind of alteration is supposed to explain (hence the $\gamma \alpha$ ó at 416b34) why perception consists in being changed and affected, and it can only do this if it presupposes an account of how alteration relates to being affected. The fact that Aristotle immediately references Generation and Corruption i 7 at 417a1-2 suggests that the 'assimilation story' found there provides that link.

We have already encountered what I take to be the key refinement in Aristotle's concept of being affected, viz., the notion of a preservative affection, or a 'preservation of that which is so potentially by that which is so actually and is like it in the way that a potentiality may be like an actuality' in contradistinction to 'a kind of destruction of something by its contrary' (417b2-5). Now it is natural, in the light of Burnyeat's analysis of the opening passages of De anima ii 5, to take this refinement of the notion of being affected to imply a refinement of the notion of being assimilated as well. But one might wish, for a number of reasons, to preserve the possibility that the way in which a perceiver is assimilated is not constrained by the way in which he is said to be affected. This option, however, is closed off in the final formulation of the 'assimilation story' at the end of the chapter. Here, Aristotle tells us that that which can perceive is affected ( $\pi \alpha ́ \alpha \chi \varepsilon \iota)$ by the sense object by becoming like it, immediately after telling us

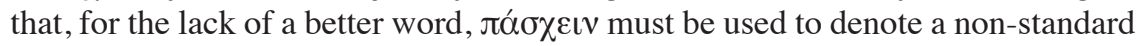
sense of being affected defined in the chapter:

Since the difference between [the sense in which a boy is a potential general and the sense in which an adult is a potential general] has no name, although it has been determined that they are different and how they are so, we must use $\tau$ ò

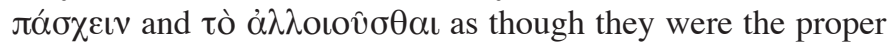
words. That which can perceive is, as we have said, potentially such as the object of perception already is actually. It is not like the object, then, when it is being affected by it ( $\pi \alpha ́ \sigma \chi \varepsilon \iota)$, but once it has been affected it becomes like it and is such as it is. ${ }^{11}$ (DA ii 5.418a1-6)

Since the only non-standard sense of being affected in sight is that of a preservative affection at $417 \mathrm{~b} 2-5$, it appears that the way in which a perceiver is said to be affected at 417b2-5 puts a constraint on how we must understand the concept of assimilation in De anima ii 5. A preservative affection, then, is not only a preservative alteration, but also a preservative assimilation. But how should we understand such a thing? The opposition of 'preservation' to 'destruction' at 417b2-5 makes it clear that an exclusive disjunction is implied: being affected is either 'a kind of destruction of something by its contrary' or a 'preservation of

11 Translations of DA ii 5 are, with minor modifications, from Hamlyn 1968. 
that which is so potentially', but not both. So at a minimum, a preservative assimilation must be an assimilation that does not involve 'a kind of destruction of

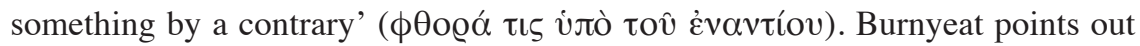
that transitions from merely having knowledge to contemplating knowledge satisfy this restriction because the termini of these changes are marked by the same word 'knows'. Likewise, transitions from merely having the faculty of perception to using the faculty of perception satisfy this restriction because the termini of these changes are marked by the same word 'perceives'. What we should take from this, claims Burnyeat 2002, 55, is that (a) the termini of preservative assimilations are not opposed, and (b) while both termini satisfy the same predicate F, one is potentially $\mathrm{F}$ while the other is actually F. Burnyeat 2002, 55-56 insists that $\mathrm{F}$ is in the category of quality, as well he should, since if it were not, then an assimilation effected by becoming $\mathrm{F}$ would not be able to play the role it is supposed to play in the opening lines of the chapter, viz., explaining the connection between being altered and being affected. Aristotle would, rather, merely be stretching the sense of the term 'alteration' to apply to changes in the category of

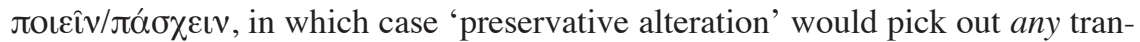
sition from having a psychological faculty to using it. In which case, there is nothing unusual or peculiarly perceptual about quasi-alteration (as Everson 1997, 93 and Sisko 1998, 336 recognize). But if $\mathrm{F}$ is in the category of quality, (a) and (b) are two horns of a dilemma. If the assimilation in question has termini that are not opposed to one another, i.e., if the terminus a quo and terminus ad quem of this transition are not dissimilar, then it is impossible to characterize it as an assimilation of the perceiver to the perceived. We should expect from any sort of assimilation, if it is to be an assimilation at all, an initial dissimilarity, and therefore opposition of termini. On this interpretation, the set of preservative assimilations is empty. Moreover, since alteration presupposes assimilation, as Burnyeat has so well argued, by denying the most basic requirement for this assimilation story to apply - an initial dissimilarity of termini-, Aristotle will have undermined his identification of the activation of the sense faculty with a kind of alteration in De anima ii $5 . .^{12}$

It is no help claiming that a thing can be assimilated by becoming $\mathrm{F}$ in a sense that is different from the way in which ordinary things become F, since any sense of being F, no matter how exotic, will be opposed to its not being $\mathrm{F}$ in that sense. Yet if I take into account Burnyeat's claim that while both termini satisfy the same predicate $\mathrm{F}$, one is potentially $\mathrm{F}$ while the other is actually $\mathrm{F}$, we land in another, equally unattractive predicament, since, though being potentially $\mathrm{F}$ and being actually $\mathrm{F}$ is an opposition, it is one that every qualitative assimilation

12 Moreover, if Aristotle were to 'go on using the language of alteration' (Burnyeat 2002, 57) and 'assimilation' while denying the most basic requirement for its correct application, then one would have to call this sort of talk misleading rather than 'refined', as Burnyeat claims, and Aristotle will have, in substance, "cut the links with the dialectic of De Generatione et Corruptione I 7 and the categorial analysis of change in Physics III 1-3' that make his psychology 'the crowning achievement of his physics' (Burnyeat 2002, 36, 58, and 78). 
shares according to Physics iii 1-3. So instead of being empty, the set of preservative assimilations is just the set of qualitative assimilations, and the 'refinements' of De anima ii 5 do no work.

At any rate, Burnyeat's grounds for insisting that the switch from having a perceptive faculty to using it is in the category of quality are weak. This sort of change is in the category of quality, he claims, because dispositions (" $\varepsilon \xi \varepsilon\llcorner\varsigma)$ such as knowledge and the faculty of sight are in the category of quality (Burnyeat $2002,55)$. But changes involving dispositions are in the category of quality because they involve the acquisition or loss of dispositions, not because they involve their activation. ${ }^{13}$ What we should infer from this, however, is not that

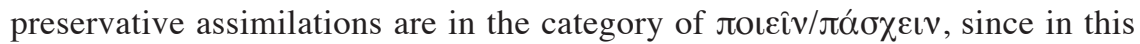
case, they would no longer be able to explain the connection between being altered and being affected. What this shows, rather, is that we should not model our understanding of preservative assimilation on the transition from having knowledge to contemplating it.

The way out of this dilemma, I suggest, is to recognize that $417 \mathrm{~b} 2-5$ contrasts preservative affections with alterations that proceed between contraries ( $\tau \grave{\alpha}$

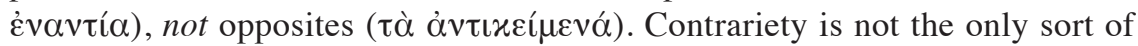
opposition the termini of a change can have. In Physics viii 7.261a32-33, Aristotle says that though all changes except circular motion proceed between opposites, not all changes proceed between contraries. And though he says in Physics i 7 that all changes proceed to a form from a privation of that form, at Metaphysics $\mathrm{x} 4.1055 \mathrm{~b} 15-16$ Aristotle claims that though every contrary is a privation, not every privation is a contrary because 'that which is deprived may be deprived in several ways'. I propose that in De anima ii 5, Aristotle is identifying the activation of the sense faculty with an alteration that lacks contrariety but not opposition of termini because it is a transition to a form from a privation of that form that is not the form's contrary.

Changes in the category of substance fit this description because substances have no contrary. ${ }^{14}$ But apart from a few odd exceptions, ${ }^{15}$ one will be hard

${ }^{13}$ Categories 8 lists four sorts of quality: states and conditions, natural capacities and incapacities, affective qualities ( $\pi \alpha \theta \eta \tau \iota x \alpha \grave{~ \pi o เ o ́ \tau \eta \tau \varepsilon \varsigma), ~ a n d ~ s h a p e, ~ a n d ~ A r i s t o t l e ~ i n ~ m a n y ~ p l a c e s ~ d e s c r i b e s ~}$ $\dot{\alpha} \lambda \lambda$ oíwoıs as change in quality (Cat. 14.15b12; Phys. viii 7.261a22; Cael. i 3.270a27; GC i 4.319b33, 320a25; Meta. xiv 2.1088a32). In Physics vii 3, however, Aristotle restricts the term $\dot{\alpha} \lambda \lambda$ oí $\omega \sigma \iota \varsigma$ to apply to changes in affective qualities, and denies that changes in states and conditions

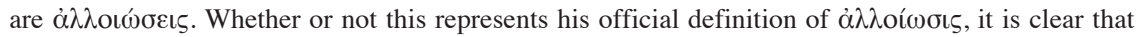

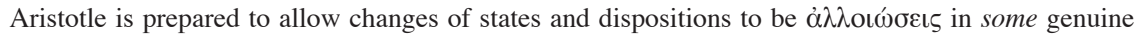

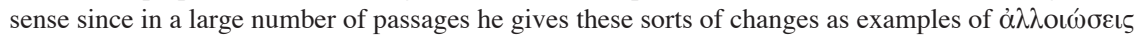
and at $G C$ i $4.319 \mathrm{~b} 12-14, \mathrm{~b} 25-31$, he even does this in the context of defining $\dot{\alpha} \lambda \lambda$ oí $\omega \sigma \mathrm{s}$. See also Cat. 5.4a31-32; Phys. iii 3.202a32-36, v 1.224a30, v 6.230a21-22, b3-5, viii 3.253b23-27, 5.257b4-6; $G C$ i 6.334a11-12; Cael. iv 3.310b17-19; Meta. viii 1.1042a36-b1, xi 6.1063a35-b1.

${ }^{14}$ Aristotle often says, substance has no contrary. See Cat. 5.3b24-25, Phys. v 2.225b10-11, Meta. xi 12.1068a11, xiv 1.1087b2-3. Philoponus In DA 144,22-25 and Damascius In Phaedonem 383,1-2 report that Aristotle says the soul has no contrary in his lost dialogue the Eudemus, and since the soul is a substance, this falls under the doctrine that substance has no contrary.

${ }^{15}$ E.g., at Cat. 5.3b31-32, 5b11-12, Aristotle says that particular quantities like 'four foot' have 
pressed to find anything in the category of quality that lacks a contrary. Barring this option, then, what we need is an alteration to a quality that has a contrary from a privation of that quality that is not its contrary, and Metaphysics x 5 seems to suggest just this possibility. In this chapter, Aristotle calls the neutral state of being neither of two contraries in a thing that is receptive of those contraries a

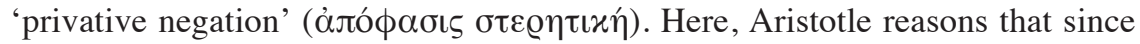
we normally ask 'whether' a quantity is greater than, less than, or equal to another, being equal to another quantity must be incompatible both with being less than it and being greater than it. And if equality is incompatible with both of these properties, it must be opposed to both of them in some way. But, since 'one thing cannot have more than one contrary' (Meta. x 4.1055a19-20), equality cannot be contrary to both. But neither can it be contrary to one but not the other, 'for why should it be contrary to the greater rather than the lesser?' (1056a5-6). It remains, then, for 'the equal' to be opposed to both 'the greater' and 'the lesser' as either a negation or as a privation (1056a15-16). 'The equal' is certainly a negation, insofar as it is neither 'the lesser' nor 'the greater', but it cannot be simply a negation, because if this were so, everything would be such that it is either equal or unequal, but only quantities can have these properties (1056a20-24), so it must also be a privation in that it is 'determinate or taken along with its receptive material' (1055b7-8). The equal, Aristotle concludes, is opposed to both 'the lesser' and 'the greater' not as a contrary, but as a 'privative negation' (1056a1718). So Aristotle implies that when a thing changes from having a quantity that is equal to another to having a quantity that is either greater than or less than another, or when a thing changes from having a quantity that is either greater than or less than another to having a quantity that is equal to another, it proceeds from a form to a privation or from a privation to a form, respectively, where the form and privation are not also contraries. The fact that Aristotle immediately (at 1056a24-27) applies his concept of a privative negation to what is neither good nor bad and to what is neither black nor white suggests that an analogous principle should hold for changes in the category of quality: An alteration from a privative negation, viz., a neutral state of being neither of two qualitative contraries $\mathrm{F}$ or $\mathrm{G}$ in a thing that is receptive of these contraries, to a state of being either $\mathrm{F}$ or $\mathrm{G}$ is an alteration to a quality from a privation of that quality that is not the quality's contrary.

Burnyeat contends that the refinement to the notion of being affected at 417b25 , and by implication, to the notions of being altered and assimilated, suffices to make them inapplicable to anything but perceptual events, and suffices for them to be so named only by courtesy. It is plain to see, however, that my interpretation of a preservative affection has neither implication, since the ordinary and obviously non-perceptual alteration caused by putting blue dye into some water is an alteration to a quality from a privation of that quality that is not the quality's

no contrary, and at Top. iv 3.123b35-37 he says that a particular disease like ophthalmia lacks a contrary, though presumably complete magnitude and disease in general do not. 
contrary. The notion of a privative negation also has an obvious application to the sense organs, since Aristotle claims that the normal condition of the sense organs is a neutral state of being neither of two contraries which the organ is receptive of. The normal state of the eye, for instance, is being neither white nor black (424a8), ${ }^{16}$ and the normal state of the organ of touch is being neither hot nor cold (424a10) and neither dry nor moist (422b6). In the case of the eye, this amounts to being colorless (418b27), while in the case of the organ of touch it amounts to being in a mean state $(424 \mathrm{a} 4,435 \mathrm{a} 21-22)$ but since each is a state of being neither of two contraries in a thing that is receptive of those contraries, the normal state of a sense organ is a privative negation. This, presumably, is why at Physics viii 7.261b19-20, Aristotle claims that the mean, like the equal, is the opposite, but not the contrary of both 'that which surpasses it and of that which it surpasses'. So the taking on of a form by a sense organ, when described non-accidentally, ${ }^{17}$ will, in normal circumstances, proceed from a privative negation. The organ of touch will proceed from states of being neither hot, nor cold, nor wet, nor dry to states of being hot, cold, wet, or dry, and the eye will proceed from a state of colorlessness to a state of being colored (in what sense hot, cold, wet, dry, and colored I leave undecided).

Lorenz considers something like this when he entertains the possibility that a change in the eye jelly from transparency to having a color might exemplify a quasi-alteration because, in such an alteration, a color is gained but not destroyed. ${ }^{18}$ But he rejects this notion of quasi-alteration on the grounds that it is not general enough. Since Aristotle's claims in De anima ii 5 are about perception in general, the definition of quasi-alteration must apply to all of the senses. And this definition of quasi-alteration cannot apply to touch, argues Lorenz, because in coming to sense hot, cold, wet, or dry, a mean state clearly is destroyed. Though this may be true, the destruction of a mean state considered as

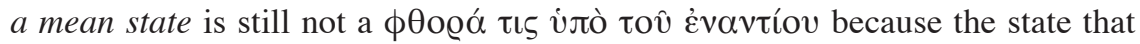
destroys it is not its contrary. The mean may be considered as a contrary of that which surpasses it and of that which it surpasses (Phys. v 1.224b30-35), but then,

16 Aristotle is being careless here because the eye is not only neither white nor black, but neither white nor black, nor grey nor any other color, viz., colorless (cf. Meta. i 8.989b8-9; Aristotle admits as much at DA ii 11.422b31-32.). At Meta. x 5.1056a27-30, he says that intermediate colors such as grey and yellow are also neither white nor black. Cf. Murphy 2006, 318, who cites Lloyd 1966, 92-93 for the claim that this is consistent with Greek usage.

17 The taking on of a form by a sense-organ may have the $x \alpha \tau \grave{\alpha} \sigma u \mu \beta \varepsilon \beta \eta x$ ó $\varsigma$ description of being a transition from the sense-organ being qualified by one form to immediately being qualified by another. But since the normal state of the sense organs is neutral, and neutrality is a necessary condition for sensation, we may infer that a $\varkappa \alpha \theta^{\prime} \alpha \dot{v} \tau$ ó description of the quasi-alteration in the sense organ is from a state of neutrality. This is confirmed in the case of touch by the fact that what we sense is the difference between the normal state of the organ of touch and the state when it is affected. This is confirmed in the case of sight by the fact that the eye jelly is intrinsically colorless, and is affected by taking on a $x \alpha \tau \grave{\alpha} \sigma v \mu \beta \varepsilon \beta \eta x o ́ \varsigma$ and $\alpha \lambda \lambda$ ó $\varrho ı$ so color.

18 Lorenz 2007, 187n17. In the scenario he is considering, Lorenz supposes that the color acquired is an ordinary color, but the literal or non-literal status of the color makes no difference to my argument. 
as Metaphysics x 5 makes clear, it is no longer considered as a mean. Again, the mean, considered as a mean, is not the contrary of that which surpasses it and of that which it surpasses, and it is essential to consider the mean temperature of the tactile organ as a mean in order to view it from the level of abstraction that is appropriate for a functioning sense organ. Otherwise it may appear that 'neutrality' will mean something different in the case of touch than it does in the case of sight (as Murphy 2005, 319-320 suggests). To take a neutral temperature to be a quality in the same range as hot and cold is to view neutrality on a material, i.e., non-functional level. Viewed from the proper functional level of abstraction, there is a single sense of neutrality, viz., privative negation, that applies to both touch and sight. Though it is true that a state of being neither hot nor cold is also, from a certain perspective, positively characterizable as 'tepid', what matters, for the tactile organ qua sense organ is that its initial state is neither of the relevant extremes (hot or cold). This is why Aristotle says, pace Johansen 1998, 217, that when our tactile organ goes from a mean temperature to a hot temperature, we transition from not perceiving a temperature to perceiving a hot temperature, not from perceiving a mean temperature to perceiving a hot temperature. Describing thermal perturbations of the tactile organ as losses of intermediate temperatures is to describe it at a material level below which the distinctions of De anima ii 5 are operating.

Burnyeat 2002, 30 also considers the idea that quasi-alterations proceed from a state of neutrality in the sense organs, but argues that this cannot be Aristotle's meaning in De anima ii 5. In De anima ii 5, claims Burnyeat, Aristotle is refining a notion of alteration prior to using it in his account of the five senses, so a concern with the neutrality of the sense organs would be premature. Still, the proper question to ask is not whether Aristotle is talking about the neutrality of the sense organs in De anima ii 5, but whether what he says there can be applied to the neutrality of the sense organs. Since the main role, in Burnyeat's interpretation, of the refinement to the concept of being affected at $417 \mathrm{~b} 2-5$ is to restrict what the notion of quasi-alteration can apply to, it is fair to ask whether quasi-alteration, when refined in this way, can be applied to a change starting from a neutral state in the sense organs, and on my interpretation of this refinement it clearly can.

Now Aristotle does not think that when we see something blue, our eye jelly goes blue in the way that water does when it is dyed blue. De sensu 3 tells us that when something goes blue in this way, it is because the bodies of the dye and the water are mixed, and when the bodies are mixed, the dyed water has its own ('oıs, 439b13) color. But our eye jelly, and air or water when they function as a medium for sight, do not take on their own color when affected by something

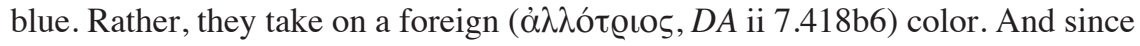
this affection involves no intermingling of matter, it seems to be strictly a change in the form of the eye jelly and the medium and not in their matter. Whether or not this foreign coloration is literal and observable by a third party is controversial. If it is a literal coloration, then it has a non-perceptual description because, according to Sorabji's interpretation of Aristotle, the sea undergoes a similar 
quasi-alteration when we move toward or away from it. ${ }^{19}$ Aristotle observes in De sensu 3.439b3-6 that the apparent color of the sea differs depending upon one's distance from it, and what this means, presumably, is that it may appear blue from a distance but transparent up close. This is not, however, what happens to our eye jelly on Burnyeat's reading. Burnyeat thinks that when something takes on a foreign color, according to Aristotle, it does so in a strictly perceptual, non-literal sense that is unobservable to third parties. My interpretation of the refinement to the notion of being affected at $417 \mathrm{~b} 2-5$ shows that De anima ii 5 need not entail this. Nor need it rule it out. In fact, it makes no difference to the way I have interpreted De anima ii 5, whether we take the relevant contraries of the quasi-alteration to be $\mathrm{F}$ and $\mathrm{G}$ in a literal or non-literal sense, since each neutral state is a state of being neither of these contraries, whether literal or non-literal, in a thing that is receptive of them. Hence, the normal state of a sense organ, on either a literal or a non-literal interpretation of $F$ and $G$, is a privative negation. I say this because I do not wish to argue whether or not there are non-literal alterations and whether the sense organs undergo them. Rather, I now wish to argue that, even if the quasi-alterations in the sense organ are non-literal in the way that Burnyeat says they are, they still admit description in non-perceptual terms.

Quasi-alteration in the sense medium and quasi-alteration in the sense organ

Burnyeat 1995b, 427, 429, 431; 2001, 133, 147, 150; 2002, 75 readily admits that, according to Aristotle, the medium and the sense organ undergo the same effect. In fact, he claims that the only difference between the change in the senseorgan and the change in the medium is where the changes happen $(2001,150)$, and this follows from two considerations. First, each sense organ as well as its corresponding medium, are constructed out of materials that are liable to be affected by the same proper sensible. Both the eye and air are transparent, which allows them both to be affected by colors (see $D A$ ii 7.418a31-b1, 419a9-11). Both the external air and the air within the ear are immobile, which allows them both to be affected by sounds (see $D A$ ii $8.419 \mathrm{~b} 21,420 \mathrm{a} 10$ ). Second, Aristotle casts the medium as a changed changer that is changed by the proper sensible and in turn changes the sense organ. ${ }^{20}$ Since changed changers characteristically undergo the same sort of change that they impart, this also implies that the medium and the sense organ undergo the same type of change. This is particu-

${ }^{19}$ Similar not in the sense that the coloration in the eye jelly depends on distance, but in the sense that both the coloration of the sea and the coloration of the eye jelly lack the material basis of the color of the water that has been dyed blue (see Sorabji 2001, 53).

${ }^{20}$ See $D A$ ii 7.419a13-15, ii 8.420a3-5, iii 12.434b26-435a5; cf. also ii 7.419a14-15, a18, Sens. 3.439a17, Insomn. $2.459 \mathrm{a} 28 \mathrm{ff}$. Note that the involvement of a changed changer need not imply that the propagation of the change through the changed changer takes time, even in the case of locomotion. Physics vii 1 shows that the transmission of locomotion through moved movers need not take time, since even an infinite series of moved movers can 'move at the same time' (242b50-51). If one moves something by means of a stick, e.g., the hand, the stick, and whatever the stick moves all move at the same time (see Phys. viii 5). 
larly clear at De anima iii $12.434 \mathrm{~b} 29 \mathrm{ff}$., where Aristotle implies that an altered alterer in the case of perception is on all fours with a moved mover in the case of local motion, the paradigmatic case in which a changed changer undergoes the same type of change as it imparts. ${ }^{21}$

Aristotle complicates this straightforward story with two claims that appear to conflict with one another. First, he says in De anima ii 7.419a12-13 and a25-30 that unmediated action on the sense organ by the body that has the proper sensible does not result in sensation. This seems to imply that I need to revise what I said about how the sense organ and the medium are constituted, viz., while the sense organ and the medium are affected in the same way, they are constituted to be affected by different bodies - the sense organ by the medium and the medium by the body that has the proper sensible. But then Aristotle claims in De anima iii 2 that the action of the proper sensible (e.g., sounding) and the affection of the sense (e.g., hearing) is a single event under two descriptions that is located in the perceiver. On the face of it, De anima ii 7 and De anima iii 2 each seem to say that the sense organ can be affected by only one sort of body, but they contradict each other regarding what that body is. De anima ii 7 seems to say that the sense organ can only be affected by the medium. But according to De anima iii 2, the sense organ would seem only to be affected by what has the proper sensible: Since the action of the proper sensible is also the action of what has the proper sensible, and De anima iii 2 countenances only one event as the action and affection of the perceiver and the perceived, then De anima iii 2 appears to leave no room for the medium to play the role of a changed changer. Now in the light of De anima ii $12.424 \mathrm{~b} 5-12$ that claims that the only effect a smell can have is to make something smell it, one might suppose there is a genuine inconsistency in the De anima - one line of thought that envisages the medium being affected by the proper sensible as a moved mover, and one line of thought that espouses what Broadie 1992, $145 \mathrm{ff}$. calls 'the restricted efficacy of sensibilia'. But I think there is a straightforward way to acquit Aristotle of this inconsistency. First, since at 424b12-13, Aristotle backtracks on his claim that the only effect a smell can have is to make something smell it, we may plausibly suppose that he advances the view only dialectically. ${ }^{22}$ Second, we can remove the conflict with De anima iii 2 if we take into account what Aristotle says about chains of changed and unchanged changers in Generation and Corruption i 7.324a25 ff.: It is only the

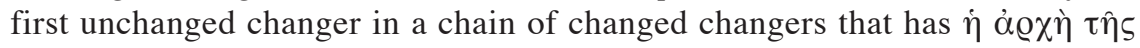

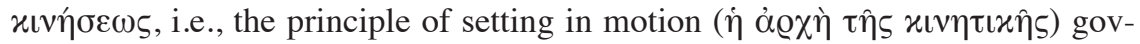
erning the change (cf. Meta. ix 8.1049b6-9). The other changers in the chain are changed by force and are, as it were, silent partners in the project of creating the

${ }^{21}$ This is entailed in the case of locomotion by the requirement in Phys. vii 2 that the moved mover and the thing moved in a forced locomotion be in contact as long as this motion is in progress, so that the moved mover must accompany the thing moved throughout its motion.

${ }^{22}$ See also Broackes 1999, 104-113, who cites passages that seem to imply that sensibilia have non-sensible effects, e.g., air takes on smells (DA ii 12), as the oil and wine (Insomn. 2.460a29), some smells may be harmful to insects ( $H A$ iv 8.20), and noises may destroy things (Cael. ii 9.290b33). 
ultimate effect. So mediation by means of changed changers does not deprive proper sensibles and the bodies that have them of their role as the proper agents of sensation by promoting the medium to this role. The proper sensible (and the body that has it) is the only changer in the chain of changed changers that has $\dot{\eta}$

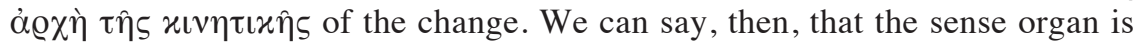
affected by the proper sensible (and the body that has it) by means of being affected by the medium, and that the effect on the medium and the sense organ is the same.

The obvious question for Burnyeat, here, is "if the medium and the eye undergo the same sort of quasi-alteration, and the medium is outside the body of the perceiver, will this sort of quasi-alteration not have a non-perceptual description?' Burnyeat clearly thinks it will not, and in the case of sight, at least, he

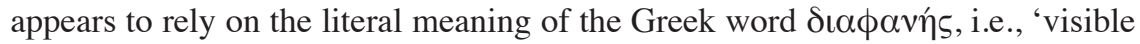
through' for his answer (as Sisko 1998, 344 suggests). Though the change in the transparent medium occurs outside the body of the perceiver, Burnyeat claims that it is nonetheless perceptual in the sense of phenomenal because it consists in a sense object becoming visible, i.e., фave@ós, through it to a perceiver. Generalizing, then, to the rest of the senses, the claim is that the effect of sense objects on perceptual media consists in sense objects becoming perceptible though them to perceivers.

But this is unsatisfyingly vague. What, precisely, does it mean for a medium to have something become perceptible through it? One idea might be to say that the media of sense only undergo a relational change - a change of coming to be spatially between a sense organ and a sense object. The change in the medium is perceptual, then, because it is the acquisition of a relation to a perceiver. While Burnyeat does claim, following Alexander, that the activation of the potentially transparent by the presence of fire is a relational change, he refrains from claiming that this is how the medium of sight, and the media of sense quite generally, are affected by the sense object. ${ }^{23}$ One obvious problem with this suggestion is that while media come to be between sense objects and sense organs all at once, not all sorts of media are said to be affected by the sense object in this manner. Transparent media, says Aristotle, are affected all at once (see, e.g., Sens. 6.446 b28-447a10), but 'he who is nearer perceives the odor sooner, and the sound of a stroke reaches us some time after it has been struck' (446a24-25). Relational change, moreover, is not the sort of change to be transmitted by changed changers. When a medium comes to be between a sense object and a sense organ, its interposition is not an affection that it receives from the sense object and passes on to the sense organ. If it is an affection at all, it is an affection of which the sense object, the medium, and the sense organ are reciprocally and simultaneously both agents and patients.

23 See, e.g., Miller 1999, 193, who unfairly attributes this view to Burnyeat and then refutes it for reasons similar to those I suggest. See also Johansen 1998, 136-145, and Price 1996, 292-293, who reject the suggestion that the change in the medium is a relational change, Price on the ground that the affection in the eye is said to linger after the sense object is gone at Insomn . 2.459b9-11. 
Perhaps, though not a relational change, the effect on the medium is, as Johansen suggests, ${ }^{24}$ something that only occurs in the presence of a perceiver. Quasi-alteration, on this view, is perceptual in the sense of perceiver-dependent. But this would mean that Aristotle misleads us in De anima ii 7 about the nature of color when he tells us that "every color is capable of setting in motion the actually transparent', at least by itself, because he should have said that color can only do this in the presence of a perceiver. Or rather, perhaps we should say that he misleads us about the nature of the actually transparent, if to be actually transparent entails not just being illuminated, but also being looked through by a perceiver. And if so, he also misleads us about the nature of the potentially transparent, which applies not just to unilluminated air, but also to illuminated air in the absence of a perceiver. And these matters would need to have slipped his mind not only in De anima ii 7, but also in De Sensu 3.439a17-b17, the other major discussion of transparency, as well as in the sixteen or so other passages where transparency is mentioned in the corpus. ${ }^{25}$ It is difficult to believe, I think, that Aristotle would have been so consistently careless. The notion, moreover, that the effect on the medium occurs only in the presence of a perceiver runs into a serious problem with the other distance senses since their media are not affected all at once. Broackes 1999, 110 points out that in the case of these media, Johansen's interpretation runs the risk of countenancing backward causation. For example, if a smell sets out from the cheese toward one's nose but one then runs away before it arrives, is the air retroactively not affected by the cheese? It seems that it would need to be if the quasi-alteration in the medium only occurs in the presence of a perceiver.

Still another idea, canvassed by Johansen and Broadie, is to claim that the effect of the proper sensible on the medium is the acquisition of a disposition to produce certain effects in a functioning sense organ. ${ }^{26}$ Since the disposition that the medium takes on is defined in terms of an effect produced on perceivers, the acquisition of this disposition by the medium is definitionally tied to perception. But at least three problems arise from this view. ${ }^{27}$ First, since the sense organ does not also acquire a disposition to produce certain effects in a functioning sense organ, this would contradict Aristotle's implication that the effect on the medium is the same as the effect on the sense organ..$^{28}$ Second, as Broadie points

${ }^{24}$ Johansen 1998, 146. Broadie 1992, 152 also considers the possibility that perceptual media are only affected in the presence of perceivers but discards it as absurd.

${ }^{25} G C$ i $4.319 \mathrm{~b} 23$, i $8.324 \mathrm{~b} 30,326 \mathrm{~b} 13 ; D A$ ii $9.421 \mathrm{~b} 31-32$, iii $1.425 \mathrm{a} 2$, iii $13.435 \mathrm{~b} 22$; Sens. $2.438 \mathrm{a} 14,438 \mathrm{~b} 6,4.442 \mathrm{a} 26,5.442 \mathrm{~b} 30,445 \mathrm{a} 13 ; H A$ viii $14.599 \mathrm{a} 27 ; P A$ ii $8.653 \mathrm{~b} 27$, ii $10.656 \mathrm{~b} 2 ; G A \mathrm{v}$ $1.780 \mathrm{a} 3,780 \mathrm{a} 27$.

26 Johansen 1998, 273-274 takes Aristotle's claim that air is affected by a smell by becoming odorous at $D A$ ii $12.424 \mathrm{~b} 16$ to suggest this doctrine. Broadie 1992, 153 considers this possibility, but discards it, due to the second objection raised below.

${ }^{27}$ I leave aside the controversial issue of whether, for Aristotle, sensible qualities are such dispositions or whether they have such dispositions (on this see Everson 1997, 114-115).

${ }^{28}$ In this case, the disposition in question would need to be disjunctive, i.e., a disposition to produce either certain effects in a functioning sense organ or to make other things have the same disposi- 
out, if the medium acquires a disposition to produce certain effects in a function-

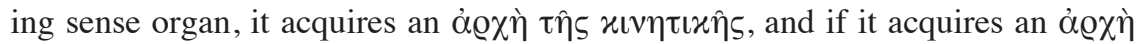

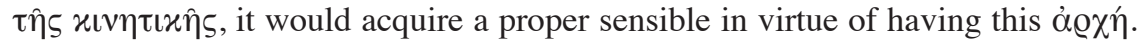
Instead of facilitating the perception of other objects, the media would become sense objects themselves. But this runs afoul of Aristotle's doctrine that unmediated action on the sense organ by the body that has the proper sensible does not result in sensation ( $D A$ ii $7.419 \mathrm{a} 12-13$ and a25-30). A third objection arises from the natural assumption that the disposition producing the effect on the medium is the same as the disposition producing the effect on the sense organ. If this were the case, it would make not only the change in the medium perceptual, but also any ordinary perceivable change in a body. The ripening of an apple would become a perceptual change on this account because it would be the acquisition of this sort of a disposition. But by 'perceptual' we do not mean to include 'perceptible'. This casts too wide a net.

Though meant to be a straightforward interpretation of how the effect of sense objects on their media can be perceptual, the hypothesis that this effect consists merely in having sense objects become perceptible through them runs into serious difficulties when we try to make it more precise. Commentators, then, have yet to show how a phenomenal interpretation of quasi-alteration in the medium can be cashed out in a way that is philosophically plausible and consistent with the texts.

\section{Conclusion}

Burnyeat is right to infer that if quasi-alterations are not ordinary alterations,

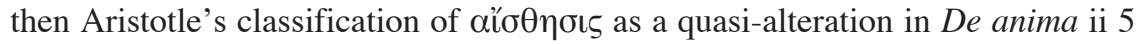
would at least have the effect of excluding ordinary alteration from the definition of $\alpha$ lo $\theta \eta \sigma ı \varsigma .{ }^{29}$ Burnyeat argues that this conclusion is unavoidable because Aristotle's account of quasi-alteration in De anima ii 5 is inconsistent with ordinary alteration, and inconsistent on two principal grounds; first, on the ground that quasi-alteration is not 'a kind of destruction of something by its contrary', and second, on the ground that quasi-alteration is only describable in perceptual terms. Others have argued, in addition, that Aristotle's account of quasi-alteration is inconsistent with ordinary alteration on the ground that quasi-alteration must consist in an activity rather than a change. I have shown, however, that each of these grounds is defeasible. We need not accept that if an alteration does not involve a destruction of something by a contrary, it cannot be an ordinary alteration because an alteration from a privative negation can be an ordinary alteration, as in the case of the coloration of a transparent substance. Nor need we conclude, it seems, that quasi-alteration is not an ordinary alteration because it is

tion.

29 This, I take it, is what is validly inferrable from Burnyeat's interpretation of quasi-alteration and the fact that Aristotle classifies $\alpha{ }^{\prime} \sigma \theta \eta \sigma \iota \varsigma$ as a quasi-alteration. Burnyeat also denies that ordinary alteration might still accompany $\alpha$ l' $\sigma \theta \eta \sigma ı \varsigma$, but Caston 2004, 265 ff., argues convincingly that he proves too much. 
perceptual. The reason is that even if quasi-alteration in the sense organ is a perceptual alteration, it is evidently not perceptual in a way that excludes a non-perceptual description, since the very same quasi-alteration occurs in the medium as

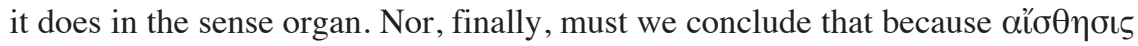

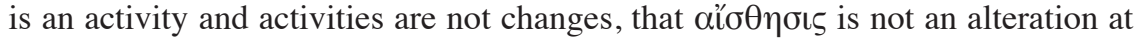
all, much less an ordinary alteration, because what Aristotle identifies with

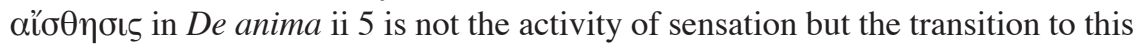
activity. Since, then, Aristotle's concept of quasi-alteration is consistent with ordinary alteration, we cannot rule out the possibility that ordinary alteration plays an essential or constitutive role in $\alpha$ lo $\sigma \theta \eta \sigma ı s$, at least on the basis of $D e$ anima ii 5. Whether or not this is a mere possibility I leave as an open question and as a topic for another occasion.

Department of Philosophy

University of California, Santa Cruz

Santa Cruz CA 95064

\section{BIBLIOGRAPHY}

Barnes, J. 1971/72. 'Aristotle's Concept of Mind' Proceedings of the Aristotelian Society 72: 101114.

Bowin, J. 2011. 'Aristotle on Various Types of Alteration in De Anima II 5' Phronesis 56: 138-161.

Broadie, S. 1992. 'Aristotle's Perceptual Realism' 137-159 in John Ellis ed. 1992. Ancient Minds. Spindel Conference. Southern Journal of Philosophy 31 suppl.

Broackes, J. 1999. 'Aristotle, Objectivity and Perception' Oxford Studies in Ancient Philosophy 17: $57-113$.

Burnyeat, M.F. 1984. Notes on Eta and Theta of Aristotle's "Metaphysics": A Study Guide. Oxford: University of Oxford Faculty of Philosophy.

Burnyeat, M.F. 1995a. 'Is an Aristotelian Philosophy of Mind Still Credible? (A Draft)' 15-26 in Nussbaum and Rorty edd. 1995.

Burnyeat, M.F. 1995b. 'How Much Happens When Aristotle Sees Red and Hears Middle C? Remarks on De Anima 2. 7-8' 421-434 in Nussbaum and Rorty, edd. 1995.

Burnyeat, M.F. 2001. 'Aquinas on "Spiritual Change” in Perception' 129-153 in Perler ed. 2001.

Burnyeat, M.F. 2002. 'De Anima II 5' Phronesis 47: 28-90.

Caston, V. 2004. 'The Spirit and the Letter: Aristotle on Perception' 245-320 in Ricardo Salles ed. 2004. Metaphysics, Soul, and Ethics: Themes from the work of Richard Sorabji. Oxford: Oxford University Press.

Cohen, S.M. 1995. 'Hylomorphism and Functionalism' 57-73 in Nussbaum and Rorty edd. 1995.

Everson, S. 1997. Aristotle on Perception. Oxford: Oxford University Press.

Gill, Mary Louise. 1989. Aristotle on Substance: The Paradox of Unity. Princeton: Princeton University Press.

Hamlyn, D.W. 1959. 'Aristotle's Account of aesthesis in the De Anima' Classical Quarterly 9: 6-16.

Heinaman, R. 1990. 'Aristotle and the Mind-Body Problem' Phronesis 35: 83-102.

Heinaman, R. 2007. 'Actuality, Potentiality and De Anima II.5' Phronesis 52: 139-187.

Hicks, R.D. 1907. Aristotle, De Anima. Cambridge: Cambridge University Press.

Johansen, T.K. 1998. Aristotle on the Sense-Organs. Cambridge: Cambridge University Press.

Lloyd, G.E.R. 1966. Polarity and Analogy: Two Types of Argumentation in Early Greek Thought. Cambridge: Cambridge University Press.

Lorenz, H. 2007. 'The assimilation of sense to sense-object in Aristotle' Oxford Studies in Ancient Philosophy: 33: 179-220.

Magee, J.M. 2000. 'Sense Organs and the Activity of Sensation in Aristotle' Phronesis 45: 306-330. 
Miller, F. D. 1999. 'Aristotle's Philosophy of Perception' Proceedings of the Boston Area Colloquium in Ancient Philosophy 15: 177-213.

Modrak, D. 1987. Aristotle: The Power of Perception. Chicago: University of Chicago Press.

Murphy, D. 2005. 'Aristotle on Why Plants Cannot Perceive' Oxford Studies in Ancient Philosophy 29: 295-339.

Murphy, D. 2006. 'The Debate of Spiritualists, Structuralists, and Literalists and De anima 423b30424a10' Ancient Philosophy 26: 305-332.

Nussbaum, M.C. and A.O. Rorty edd. 1995. Essays on Aristotle's De Anima. Oxford: Oxford University Press.

Perler, D. ed. 2001. Ancient and Medieval Theories of Intentionality. Leiden: Brill.

Polansky, Ronald. 2007. Aristotle's De Anima. Cambridge: Cambridge University Press.

Price, A.W. 1996. 'Aristotelian Perceptions' Proceedings of the Boston Area Colloquium in Ancient Philosophy 12: 285-309.

Sisko, J. 1998. 'Alteration and Quasi-Alteration: A Critical Notice of Stephen Everson, Aristotle on Perception' Oxford Studies in Ancient Philosophy 16: 331-352.

Sorabji, R.R.K. 1995. 'Intentionality and Physiological Processes: Aristotle's Theory of Sense-Perception' 195-225 in Nussbaum and Rorty edd. 1995.

Sorabji, R.R.K. 2001. 'Aristotle on Sensory Processes and Intentionality: A Reply to Burnyeat' 49-61 in Perler ed. 2001. 